\title{
Qualidade pós-colheita de frutas e hortaliças cultivadas de forma orgânica
}

\author{
Fruit post-harvest quality and vegetable cultivated organic way \\ Emmanuel Moreira Pereira ${ }^{l}$, Yvana Maria Gomes dos Santos ${ }^{1}$, Manoel Tolentino Leite Filho ${ }^{l}$,Sinara Pereira Fragoso ${ }^{2}$, \\ Bárbara Bruna Maniçoba Pereira ${ }^{3}$
}

Resumo: As frutas e hortaliças movimentam uma grande parte da economia regional e nacional, a produção orgânica vem ganhando cada vez mais destaque, pela qualidade, isenta de contaminantes químicos advindos de agroquímicos. Objetivou-se nesse trabalho determinar a qualidade pós-colheita das hortaliças tipo folha (alface, couve folha e rúcula) e fruto (pimentacumari) assim como as frutas cítricas (toranja e tangerina) em diferentes estádios de maturação, produzidas de forma orgânica no brejo paraibano. O experimento foi conduzido no Laboratório de Pesquisa e Desenvolvimento de Produtos Frutohortícolas pertencente ao Centro de Ciências Humanas Sociais e Agrárias- Bananeiras/PB, pertencente à Universidade Federal da Paraíba. As características analisadas foram: Sólidos solúveis (SS), Acidez titulavel (AT), pH, Vitamina C e Condutividade elétrica (CE). Para as frutas (laranja e toranja) e hortaliça tipo fruto (pimenta), avaliou-se em diferentes estádios de maturação, em função da coloração da casca. A alface, couve e a rúcula apresentam bons teores de sólidos solúveis totais e vitamina C. Para a pimenta Cumari todos os parâmetros com exceção do pH sofreram aumentos consideráveis a medida que avança a maturação. Para tangerina e cravo necessita-se de mais estudos. A toranja apresentou bons teores de vitamina C.

Palavras-chaves: Físico-química, Folhosas, Frutos, Agricultura orgânica.

\begin{abstract}
Fruits and vegetables move a large part of regional and national economy, organic farming is gaining more prominence, quality, free of chemical contaminants coming from agroquímicos. Objetivou up this work to determine the postharvest quality of leaf type vegetables (lettuce, cabbage leaf and arugula) and fruit (pimentacumari) as well as citrus fruits (grapefruit and tangerine) at different stages of maturation, produced organically in Paraiba swamp. The experiment was conducted at the Research Laboratory and Frutohortícolas Product Development belonging to the Centre for Social and Human Sciences Agrarias- Bananeiras/PB, belonging to the Federal University of Paraíba. The analyzed characteristics were: soluble solids (SS), titratable acidity (TA), $\mathrm{pH}$, vitamin $\mathrm{C}$ and electrical conductivity (EC). For fruit (orange and grapefruit) and vegetable fruit type (pepper), evaluated at different stages of maturity, depending on the skin color. Lettuce, cabbage and arugula present good solids and total soluble vitamin C. Pepper Cumari all pH parameters with the exception suffered considerable increases as he advances ripening. To tangerine clove need to further study. The grapefruit showed good vitamin C content.
\end{abstract}

Keywords: Physical chemistry, hardwoods, Fruits, Organic Agriculture.

\footnotetext{
*Autor para correspondência

Recebido para publicação em 24/03/2015; aprovado em 25/04/2015

${ }^{1}$ Pós-graduando em Engenharia Agrícola - UFCG/CTRN - Universidade Federal de Campina Grande-PB, email: emmanuel16mop@ hotmail.com

${ }^{2}$ Pós-graduando em Ciência e Tecnologia de Alimentos - UFPB - Universidade Federal da Paraíba

${ }^{3}$ Mestre em Sistemas Agroindustriais - UFCG/CCTA - Universidade Federal de Campina Grande-PB
} 


\section{INTRODUÇÃO}

O consumo de hortaliças e frutas tem aumentado não só pelo crescente aumento da população, mas também pela tendência de mudança no hábito alimentar do consumidor, tornando-se inevitável o aumento da produção (OHSE et al., 2001).

O consumo de frutas, legumes e verduras tem sido associado à diminuição do risco de mortalidade (Agudo et al, 2007) e redução da ocorrência de doenças crônicas, tais como as doenças cardiovasculares (Dauchet et al, 2006), derrames (He et al, 2006) e alguns tipos de câncer (Key et al, 2002).

As frutas e hortaliças movimentam uma grande parte da economia regional e nacional, a produção orgânica vem ganhando cada vez mais destaque, pela qualidade, isenta de contaminantes químicos advindos de agroquímicos, caindo de vez na preferência do consumidor.

A alface (Lactuca sativa) tem grande importância na alimentação e na saúde humana destacando-se, principalmente, como fonte de vitaminas e sais minerais, e por constituir-se na mais popular hortaliça folhosa (SILVA et al, 2011).

A couve folha (Brassicaoleracea Var. acephala) pertence à família das Brassicaceae, desta família é a espécie que mais se assemelha ao ancestral, couve silvestre. A rúcula (Eruca sativa) é uma hortaliça folhosa originária da região Mediterrânea, muito popular nas regiões de colonização italiana no Brasil,rica em potássio, enxofre, ferro e vitaminas A e C, é apreciada pelo sabor picante e cheiro agradável e acentuado (TRANI E PASSOS, 1998).

O Brasil é o segundo maior produtor de pimenta no mundo, e centro da diversidade do gênero Capsicum (COSTA et al., 2010) As pimentas da espécie Capsicum apresentam expressiva importância econômica, em grande parte, por seu aproveitamento na alimentação humana, podendo ser consumidas frescas ou cozidas, imaturas ou maduras, fermentadas, desidratadas, bem como na forma de molhos e geleias, tendências da gastronomia contemporânea. Servem de corantes, aromatizantes e oleorresinas, usados em produtos alimentícios para conferir sabor e aumentar a estabilidade oxidativa dos lipídeos. (ANTUNES et al., 2012).

Dentre as espécies do gênero Capsicum, cinco são domesticadas e largamente cultivadas e utilizadas pelo homem: Capsicum annuum; C. baccatum; C. chinense; C. frutescens e C. pubescens. Destas, apenas C. pubescens não é cultivada no Brasil. O centro de origem das pimentas do gênero Capsicum é o continente americano (CARVALHO et al., 2003)

Apesar da grande importância socioeconômica das pimentas ainda existe uma grande carência de dados sobre as características físico-químicas de muitas espécies, inclusive da pimenta Cumari.

As tangerinas constituem o segundo grupo de frutos mais importantes na citricultura mundial, ocupando a maior faixa de adaptação climática entre os citros cultivados, uma vez que são plantas igualmente tolerantes a níveis altos e baixos de temperatura ambiente. Entre as cultivares mais exploradas, destacam-se a Satsuma, Mexerica, Ponkan, Dancye Cravo, além dos híbridos Murcott e Lee (FIGEIREDO, 1991). Segundo Rossi et al, (2004) a produção brasileira de tangerinas é voltada predominantemente para o mercado interno de frutas frescas, sendo a tangerineira
'Ponkan' (Citrusreticulata Blanco) a principal cultivar plantada.A tangerina 'Cravo"(Citrusreticulata Blanco)é uma fruta cítrica de cor alaranjada e sabor adocicado.

A toranja (Citrus $\times$ paradisi) é um citrino grande, parente da laranja e do limão, e é categorizada como branca (loira), rosa ou rubi. No entanto, esta terminologia não reflete a cor da sua casca, amarela ou amarelo rosado, mas descreve a cor da sua polpa.As toranjas costumam variar em diâmetro, entre 10 a 15 centímetros, tendo algumas variedades sementes, e outras não. O sabor maravilhoso da toranja é divinal, tal como o seu nome em latim, Citrusparadisi, dá a entender (Rodrigues e Guedes 2008).

Objetivou-se nesse trabalhodeterminar a qualidade pós-colheita das hortaliças tipo folha (alface, couve folha e rúcula), fruto (pimentacumari) e de frutas cítricas (toranja e tangerina),em diferentes estádios de maturação para as frutas em função das coloração da casca, produzidas de forma orgânica no brejo paraibano.

\section{MATERIAL E MÉTODOS}

\section{Material Vegetal}

O experimento foi conduzido no Laboratório de Pesquisa e Desenvolvimento de Produtos Frutohortícolas PDFRUTHO do Centro de Ciências Humanas Sociais e Agrarias, Campus Bananeiras - Bananeiras/PB, campus III, pertencente à Universidade Federal da Paraíba. Ashortaliçase as frutas foram produzidasno setor de Agricultura do CCHSA, Campus Bananeiras - Bananeiras/PB, de forma orgânica, sem a utilização de insumos agroquímicos, o manejo deu-se por meio de capinas manuais a irrigação foi feita por meio de micro aspersores sempre que havia a necessidade, o adubo utilizado foi esterco bovino, proveniente do próprio Campus III. As hortaliças e frutas foramconduzidas ao PDFRUTHO, seguindo as etapas de seleção para a retirada de partes residuais dos vegetais, limpeza com água corrente para a retirada das sujidades aderida ao material vegetal. Para as frutas e hortaliça tipo fruto, avaliou-se em diferentes estádios de maturação, em função da coloração da casca, para o estádio de maturação 1 (E1) a casca apresentava coloração totalmente verde , para o estádio de maturação 2(E2) amarelo esverdeado e para o estádio de maturação 3 (E3) vermelho alaranjado.

\section{Determinações Químicas}

As determinações foram feitas em triplicatas e obtidas as médias, seguindo o desvio padrão (Dp).As determinações foram realizadas, conforme descreve as metodologias do IAL (2008) opH conforme a AOAC(2005).

\section{- Sólidos Solúveis (SS)}

O suco celular foi extraído a partir de $100 \mathrm{~g}$ de material vegetal, triturados com auxílio de um extrator de suco celular. O teor de sólidos solúveis totais foi lido em um refratômetro digital com compensação automática de temperatura.

\section{- Acidez Titulável (AT)}

A acidez foi medida em $5 \mathrm{~mL}$ de suco, homogeneizado em $45 \mathrm{~mL}$ de água destilada. A solução contendo a amostra foi titulada com $\mathrm{NaOH} 0,1 \mathrm{~N}$ até atingir o ponto de viragem do 
indicador fenoftaleína, confirmado pela faixa de $\mathrm{pH}$ do indicador de 8,2 .

\section{- Potencial Hidrogeniônico (pH)}

O potencial hidrogeniônico foi determinado no suco de acordo com o número de repetições, utilizando-se um potenciômetro digital de bancada, para estimar o teor de íons $\mathrm{H}+$.

\section{- Vitamina C}

A vitamina $C$ foi estimada por titulação, utilizando-se $5 \mathrm{~mL}$ de suco do broto de palma acrescido de $45 \mathrm{~mL}$ de ácido oxálico $0,5 \%$ e titulado com solução de Tillmans até atingir coloração rosa, conforme método (365/IV).

\section{- Condutividade elétrica (CE)}

A condutividade elétrica foi determinada no suco de acordo com o número de repetições, utilizando-se um condutivimetro digital de bancada, para estimar o teor de eletrólitos.

\section{RESULTADOS e DISCUSSÃO}

A cultura da alface apresentou valor de pH5,8, AT de $0,1 \%$ de SST de $2,0^{\circ}$ Brix, de vitamina C de $2,0 \mathrm{mg} / 100 \mathrm{~g}$ e condutividade elétrica de 13,2mS (Tabela 1), respectivamente. Menezes et. al. (2005) realizando trabalhos com alface minimamente processado obteve valores de $\mathrm{pH}$ variando de 5 a 7 , de SS variando entre 0,22 a $0,24{ }^{\circ}$ Brix e de AT em torno de $0,4 \%$, os vegetais minimamente processados sofre uma mudança na composição química em função ao processo de processamento. Moraes et. al., (2010) constatou valores de vitamina $\mathrm{C}$ para a fase final da alface de $6 \mathrm{mg} / 100 \mathrm{~g}$.

Já para a cultura da couve folha, obteve valores de pH5,6, AT0,2\%, SST de 3,7 ${ }^{\circ}$ Brix, vitamina C de $2,8 \mathrm{mg} / 100 \mathrm{~g}$ e condutividade elétrica de $13,0 \mathrm{mS}$ (Tabela1), respectivamente. Evangelista et al.,(2009) trabalhando com couve-chinesa minimamente processada encontrou valores de $\mathrm{Ph} 5,95$, Vitamina C $8,97 \mathrm{mg} / 100 \mathrm{~g}$, SST $2,7^{\circ}$ Brix e AT de $0,13 \%$, valores médios correspondentes com os encontrados neste trabalhos, divergindo apenas a vitamina $\mathrm{C}$, que poderá ter sido influenciada pela variedade da couve e o clima da região.

Para a cultura da rúcula obteve-se valores de $\mathrm{pH} 5,6$, AT0,2\%, SS de $2,4^{\circ}$ Brix, vitamina C de $2,7 \mathrm{mg} / 100 \mathrm{~g}$ e condutividade elétrica de $13,0 \mathrm{mS}$ (Tabela1), respectivamente. Em trabalhos realizados por Fabriet al., (2004) em diferentes variedades rúcula encontraram valores de AT em torno de 0,1\%, SS de $3,6^{\circ}$ Brix e de Vitamina C de 49,62 mg/100g. Para os valores de $\mathrm{pH}$ em pesquisas realizadas por Gonzales et. al., (2006) com rúcula minimamente processada determinou valores condizentes com os encontrados neste estudo.

Os dados decondutividade elétrica destas folhosas variou conforme a espécie (Tabela1). Nascimento et al., (2003) encontraram valores em hortaliças tipo folha que variavam entre 0,05 a $2,60 \mathrm{dS} / \mathrm{m}$.

Tabela 1. Valores médios $(n=3)$ e desvio padrão de $\mathrm{pH}$, vitamina $\mathrm{C}$, sólidos solúveis, acidez titulável e condutividade elétrica em hortaliças tipo folha.

\begin{tabular}{cccccc}
\hline \multirow{2}{*}{ Hortaliças } & \multicolumn{5}{c}{ Determinações químicas } \\
\cline { 2 - 6 } & $\mathrm{pH}$ & $\begin{array}{c}\text { Vitamina C } \\
(\mathrm{mg} / 100 \mathrm{~g})\end{array}$ & $\begin{array}{c}\text { SS } \\
\left({ }^{\circ} \text { Brix }\right)\end{array}$ & AT $(\%)$ & Cond. Elétrica (mS) \\
Alface & $5,8 \pm 0,1$ & $2,0 \pm 0,3$ & $2,0 \pm 0,1$ & $0,1 \pm 0,2$ & $13,2 \pm 0,4$ \\
Couve & $5,6 \pm 0,0$ & $2,8 \pm, 04$ & $3,7 \pm 0,3$ & $0,2 \pm 0,1$ & $13,0 \pm 0,1$ \\
Rúcula & $5,6 \pm 0,1$ & $2,7 \pm 0,5$ & $2,4 \pm 0,2$ & $0,2 \pm 0,1$ & $13,0 \pm 0,1$ \\
\hline
\end{tabular}

Para a pimenta Cumari os valores obtidos de $\mathrm{pH}$ nos três estádios não houve variações consideráveis de 5,11, 4,92 e 5,05 , respectivamente (tabela 2). Braga et al. (2013) obteve média de $\mathrm{pH}$ semelhante de $\mathrm{pH} 5,17 \mathrm{em}$ pimenta.

Contudo, os demais parâmetros apresentaram comportamentos similares onde, tenderam a aumentar conforme a elevação do estádio de maturação. A vitamina $\mathrm{C}$ apresentou no E1de 24,23, E2 de 42,99 e E3 de 60,64mg/100g.O SS apresentou no E1 de 5,7, no E2 de 7,3 e de $10,03^{\circ}$ Brix no E3. Para os valores de AT comportou-se no E1 de 0,23, E2 de 0,25 e E3 de 0,54\%, respectivamente (tabela2). O aumento nos teores de Vitamina C, SS e AT à medida que o vegetal chega ao ponto de maturação é um fenômeno comum na pós-colheita, uma vez que,nas reações metabólicas há síntese desses compostos com o maior grau de maturação, ficando dissolvidos no suco celular do vegetal.
Em trabalhos realizados por Braga et al. (2013) com pimenta cultivadas em Paraipaba-CE, obtiveram valores similares encontrados neste trabalho de $21,09 \mathrm{mg} / 100 \mathrm{~g}$ para vitamina $\mathrm{C}$, de $32,09^{\circ}$ Brix para SS e $0,43 \%$.

A condutividade elétrica assim como os demais parâmetros apresentou visível aumento à medida que a maturação avançano E1 de 7,02, E2 de 8,47 e E3 de 9,73 mS. Diniz et al. (2003), verificou que o nível de concentração de sólidos influencia diretamente no nível de condutividade em seu experimento com condutividade elétrica de polpas de acerola concentradas(Tabela 2).

$\mathrm{Na}$ (Tabela 2) estão representados os dados referentes à caracterização físico-química da Pimenta Cumari (Capsicum Baccatum L. Var. Baccatum) em diferentes estádios de maturação. 
Tabela 2. Valores médios $(n=3)$ e desvio padrão de $\mathrm{pH}$, vitamina $\mathrm{C}$, sólidos solúveis, acidez titulável e condutividade elétrica de Pimenta Cumari em diferentes estádios de maturação.

\begin{tabular}{lccccc}
\hline & pH & $\begin{array}{c}\text { Vit C } \\
(\mathbf{m g} / \mathbf{1 0 0 g})\end{array}$ & $\begin{array}{c}\text { SST } \\
\left({ }^{\circ} \text { Brix }\right)\end{array}$ & AT(\%) & $\begin{array}{c}\text { Cond. Eletrica } \\
(\mathbf{m S})\end{array}$ \\
\hline Estádio 1 & $5,11 \pm 0,02$ & $24,23 \pm 0,25$ & $5,70 \pm 0,26$ & $0,23 \pm 0,12$ & $7,02 \pm 0,08$ \\
Estádio 2 & $4,92 \pm 0,03$ & $42,99 \pm 0,50$ & $7,30 \pm 0,30$ & $0,35 \pm 0,15$ & $8,47 \pm 0,04$ \\
Estádio 3 & $5,05 \pm 0,02$ & $60,64 \pm 0,40$ & $10,03 \pm 0,06$ & $0,54 \pm 0,31$ & $9,73 \pm 0,03$ \\
\hline
\end{tabular}

Para a tangerina cravo no E1 os valores encontrados depHfoi de 2,6 , AT de $1,70 \%$, SS de $9,8^{\circ}$ Brix, vitamina C de $19, \mathrm{mg} / 100 \mathrm{~g}$ e condutividade elétrica de $2,7 \mathrm{mS}$ (Tabela3). Já para o E2 os valores encontrados para o $\mathrm{pH}$ de 2,6, de AT de $1,38 \%$, SS de $10,4{ }^{\circ}$ Brix, vitamina C de $19,1 \mathrm{mg} / 100 \mathrm{~g}$ e condutividade elétrica de $2,5 \mathrm{mS}$ (Tabela3). Contudo para o E3 os valores encontrados para o $\mathrm{pH}$ de 2,5, de AT de 1,81\%, SS de $10{ }^{\circ}$ Brix, vitamina $\mathrm{C}$ de $20 \mathrm{mg} / 100 \mathrm{~g}$ e condutividade elétrica de 2,6 mS (Tabela3), respectivamente. $\mathrm{O} \mathrm{pH}$ praticamente não houve variação em todos os estádios de maturação, comportamento similar pode-se observar nos valores de SS e vitamina C. Para os valores de AT e condutividade elétrica nota-se uma discrepância no E2, podendo ser atribuída ao consumo de ácidos orgânicos em reações metabólicas induzidas pelo processo fisiológico de maturação neste estádio. Corroborando com este trabalho, estudos feitos por Couto et al., (2010) com determinações analíticas em variedades cítricas observaram que o $\mathrm{pH}$ variava entre 3,2 a 5,43, de AT de 0,8 a 1,48\%, de SS 9,11 a 14,33 e vitamina $\mathrm{C}$ de 21,47 a $80,03 \mathrm{mg} / 100 \mathrm{~g}$. Valores esses que condizem com os encontrados neste trabalho, a variação em função da vitamina $\mathrm{C}$ pode ser atribuída a fisiologia e a capacidade antioxidante de cada espécie, assim como o clima a região e nutrição da planta

Tabela 3. Valores médios $(n=3)$ e desvio padrão de $\mathrm{pH}$, vitamina $\mathrm{C}$, sólidos solúveis, acidez total titulável e condutividade elétrica de Tangerina "Cravo" em diferentes estádios de maturação.

\begin{tabular}{lccccc}
\hline & $\mathbf{p H}$ & $\begin{array}{c}\text { Vit C } \\
(\mathbf{m g} / \mathbf{1 0 0 g})\end{array}$ & $\begin{array}{c}\text { SST } \\
\left({ }^{\circ} \text { Brix }\right)\end{array}$ & AT(\%) & $\begin{array}{c}\text { Cond. Eletrica } \\
(\mathbf{m S})\end{array}$ \\
\hline Estádio 1 & $2,64 \pm 0,01$ & $30,45 \pm 0,77$ & $9,87 \pm 0,02$ & $1,70 \pm 0,15$ & $2,71 \pm 0,04$ \\
Estádio 2 & $2,68 \pm 0,03$ & $29,22 \pm 0,15$ & $10,47 \pm 0,01$ & $1,38 \pm 0,06$ & $2,53 \pm 0,02$ \\
Estádio 3 & $2,56 \pm 0,01$ & $30,60 \pm 0,31$ & $10,00 \pm 0,03$ & $1,81 \pm 0,20$ & $2,63 \pm 0,03$ \\
\hline
\end{tabular}

As toranjas analisadas (Tabela 4) apresentaram valores dos estádios E1, E2 e E3, para pH de 3,75, 3,58 e 3,74, vitamina C de 24,23, 17,24 e18,51 mg/100g, SS de 9,67, 10,4 e $10,5^{\circ}$ Brix, AT de $0,48,0,58$ e $0,45 \%$ e condutividade elétrica de 3,28, 2,83 e 2,71 mS, respectivamente. Estes valores variaram conforme do tempo de maturação, passível de influência das alterações fisiológicas que são consumidoras de boa parte destes compostos analisados. Corroborando com os índices encontrados neste trabalho, em estudos realizados por Silva et al. (2005) com qualidade de suco de laranja comerciais, encontraram valores de $\mathrm{pH}$ que variaram entre 3,51 a 4,58 , de vitamina $\mathrm{C}$ de 17,25 a 62,4 $\mathrm{mg} / 100 \mathrm{~g}$, de SS 10,5 a $12,5 \%$ e AT de 0,67 a 0,88 .

Tabela 4. Valores médios $(n=3)$ e desvio padrão de $\mathrm{pH}$, vitamina $\mathrm{C}$, sólidos solúveis, acidez total titulável e condutividade elétrica de Toranja em diferentes estádios de maturação.

\begin{tabular}{lccccc}
\hline & pH & $\begin{array}{c}\text { Vit C } \\
(\mathbf{m g} / \mathbf{1 0 0 g})\end{array}$ & $\begin{array}{c}\text { SS } \\
\left({ }^{\circ} \text { Brix }\right)\end{array}$ & AT(\%) & $\begin{array}{c}\text { Cond. Eletrica } \\
(\mathbf{m S})\end{array}$ \\
\hline Estádio 1 & $3,75 \pm 0,04$ & $24,23 \pm 0,39$ & $9,67 \pm 0,01$ & $0,48 \pm 0,06$ & $3,28 \pm 0,08$ \\
Estádio 2 & $3,58 \pm 0,01$ & $17,24 \pm 0,23$ & $10,40 \pm 0,03$ & $0,58 \pm 0,20$ & $2,83 \pm 0,13$ \\
Estádio 3 & $3,74 \pm 0,01$ & $18,51 \pm 0,15$ & $10,50 \pm 0,01$ & $0,45 \pm 0,06$ & $2,71 \pm 0,24$ \\
\hline
\end{tabular}

\section{CONCLUSÕES}

- As hortaliças tipo folha são de grande importância na dieta e nutrição humana. A alface, couve e a rúcula apresentam bons teores de sólidos solúveis totais e vitamina $\mathrm{C}$, essenciais diariamente na alimentação humana;

- Para a pimenta Cumari todos os parâmetros com exceção do $\mathrm{pH}$ sofreram aumentos consideráveis a medida que avança a maturação, em consequência das transformações fisiológicas que ocorrem no fruto decorrentes da maturação o estádio 3 apresentou perfeita condição para o consumo;

- Paraa tangerina cravo necessita-se de mais estudos, com o intuito de aprofundar os conhecimentos das características físico-químicas, no entanto, apresenta-se como um alimento saudável e rico em vitamina $C$;

- A toranja apresentou bons teores de vitamina C, contudo, faz-se necessário uma determinação do potencial toxicológico. 


\section{REFERÊNCIAS BIBLIOGRÁFICAS}

AGUDO, A.; CABRERA, L.; AMIANO, P.; ARDANAZ, E.; BARRICARTE, A.; BERENGUER, T. Fruit and vegetable intakes, dietary antioxidant nutrients, and total mortality in Spanish adults: findings from the Spanish cohort of the European Prospective Investigation into Cancer and Nutrition (EPIC-Spain). Am J ClinNutr; 85:1634-42. 2007.

ANTUNES, M. A.; VANZELA, E. S. L.; CHAVES, J. B. P.; RAMOS, A. M.; STRINGHETA, P. C.; FERNANDES, P. É.Controle de qualidade de produtos à base de pimenta. Infor $\neg$ meAgropecuário, Belo Horizonte, v. 33, n. 267, p. 41-51, mar./abr. 2012.

AOAC - Association Of Official Analytical Chemists. Official methods of analysis of aoac international.17th ed. Gaithersburg. 2005.

BRAGA, T. R., PEREIRA, R. C. A., SILVEIRA, M. R. S., SILVA, L. R., SILVA, A. R., \& OLIVEIRA, M. M. T. Caracterização físico-química de progênies de pimentas cultivadas em Paraipaba-CE. Embrapa Agroindústria Tropical-Artigo em periódico indexado (ALICE).2014.

CARVALHO, S. I. C. ; BIANCHETTI, L. B.; BUSTAMANTE, P. G.; SILVA, D. B. Catálogo de germoplasma de pimentas e pimentões (Capsicum spp.) da Embrapa Hortaliças. Brasília: Embrapa Hortaliças. doc. 49. p. 49.2003.

COSTA, L. M., MOURA, N. F., MARANGONI, C., MENDES, C. E., OLIVEIRA TEIXEIRA, A. Atividade antioxidante de pimentas do gênero Capsicum.2010.

COUTO, MEYLENE AL; CANNIATTI-BRAZACA, SOLANGE G. Quantificação de vitamina $C$ e capacidade antioxidante de variedades cítricas. Ciência e Tecnologia de Alimentos, v. 30, n. Supl1, p. 15-19. 2010.

DAUCHET. L.; AMOUYEL, P.; HERCBERG, S.; DALLONGEVILLE, J. Fruit and vegetable consumption and risk of coronary heart disease: a meta-analysis of cohort studies. JornalNutrition; 136:2588-93. 2006.

DINIZ, E.; FIGUEIREDO, R. M. F.; QUEIROZ, A. J. M. Atividade de água e condutividade elétrica de polpas de acerola concentradas. Revista Brasileira de Produtos Agroindustriais, Campina Grande, v. Especial, n.1, p. 9$17 . \quad$ Disponível em: <http://www.deag.ufcg.edu.br/rbpa/rev5e/Art5e2.pdf > Acesso em: 28mar. 2015.2003.

EVANGELISTA, R. M., VIEITES, R. L., CASTRO, P. S. D., RALL, V. L. M. Qualidade de couve-chinesa minimamente processada e tratada com diferentes produtos. Ciência e Tecnologia de Alimentos, 2(29), 324332.2009 .

FABRI, E. G., SALA, F. C., FABRÍCIO, F., RONDINO, E., MINAMI, K., COSTA, C., JACOMINO, A. Avaliação da qualidade variedades de rúcula. Horticultura Brasileira, Brasília, 22(2).2004

FIGUEIREDO, J. O. Variedades-copa de valor comercial. In: RODRIGUES, O.; VIEGAS, F., POMPEU, JR., J.; AMARO, A. A. (eds.) Citricultura brasileira. Campinas: Fundação Cargill, p. 228-264.1991.
GONZALEZ, A. F., AYUB, R. A., REGHIN, M. Y. Conservação de rúcula minimamente processada produzida em campo aberto e cultivo protegido com agrotêxtil. HorticulturaBrasileira, 24(3), 360-362. 2006.

HE FJ, NOWSON CA, MACGREGOR GA. Fruit and vegetable consumption and stroke-meta-analysis of cohort studies. Lancet; 367:320-6.2006.

INSTITUTO ADOLFO LUTZ. Métodos físico-químicos para análise de alimentos. $4^{\mathrm{a}}$ ed. $1^{\mathrm{a}}$ ediçãodigital.São Paulo: IMESP. 2008.

KEY, T. J.; ALLEN, N. E.; SPENCER, E. A.; TRAVIS, R. C.The effect of diet on risk of cancer.Lancet; 360:861-8. 2002.

MENEZES, E. M., FERNANDES, E. C., SABAA-SRUR, A. U. Folhas de alface lisa (Lactuca sativa) minimamente processadas armazenadas em atmosfera modificada: análises físicas, químicas e físico-químicas. Ciencia e Tecnologia de Alimentos, 25, 60-62.2005.

MORAES, F. A., COTA, A. M., CAMPOS, F. M., PINHEIRO-SANT'ANA, H. M. Perdas de vitamina C em hortaliças durante $\mathrm{o}$ armazenamento, preparo e distribuição em restaurantes. Ciência Saúde Colet, 15(1), 51-62.2010.

NASCIMENTO, W. M., SILVA, J. B., CARRIJO, O. A. Germinação de sementes de hortaliças em diferentes substratos para produção de mudas. Horticultura brasileira, 21(2), 311.2003.

OHSE, S., DOURADO-NETO, D., MANFRON, P. A., SANTOS, O. S. D. Qualidade de cultivares de alface produzidos em hidroponia. ScientiaAgricola, 58(1), 181185.2001

RODRIGUES, S. M. GUEDES, C. F. R. Os Alimentos Mais Saudáveis do Mundo: TORANJA, Acessado em: 30/05/2015. Disponível em: http://www.alimentacaosaudavel.org/toranja.html. 2008;

ROSSI, E. P.; LIMA, L. C. O.; SOUZA, H. A.; SILVA, M.; MENDONÇA, V.; RAMOS, J. D. Efeito do raleio químico nas características físico-química dos frutos de tangerineira 'ponkan'. XIII Congresso dos PósGraduandos da UFLA. 14 a 17 de setembro de 2004.

SILVA, E. M. N. C. P; FERREIRA, R. L. F; ARAÚJO, N. E. T. O. S. E; TAVELLA, L. B; SOLINO, A. J. S. Qualidade de alface crespa cultivada em sistema orgânico, convencional e hidropônico. Horticultura Brasileira 29: 242-245. 2011.

SILVA, P. T., FIALHO, E., LOPES, M. L. M., VALENTEMESQUITA, V. L. Sucos de laranja industrializados e preparados sólidos para refrescos: estabilidade química e físico-química. Ciência e Tecnologia de Alimentos, 25(3), 401-622. 2005.

TRANI PE; PASSOS FA. Rúcula (pinchão). In: FAHL JL; CAMARGO MBP; PIZINATTO MA; BETTI JA; MELO AMT; DEMARIA IC; FURLANI AMC (eds). Instruções agrícolas para as principais culturas econômicas. Campinas: IAC. p. 241-242. (IAC. Boletim, 200).1998. 